\title{
interview
}

\section{Flying qubit carrying a spin qubit}

\author{
Complete quantum control of an electron spin qubit can be achieved using a long-lived quantum-dot \\ spin qubit coupled to a GaAs-based photonic-crystal cavity. Samuel Carter from the Naval Research \\ Laboratory says that this system could be used as a node in a quantum network.
}

What is your work about?

A major concern in the field of quantum information processing has been how to move from a single qubit to multiple qubits. In particular, it is challenging to produce the interaction between two qubits separated by a significant distance. Our approach involved combining ideas from different fields such as quantum optics, semiconductor physics and nanophotonics. Amalgamating these ideas also required combining different physical systems, namely spin qubits in self-assembled quantum dots and photonic-crystal cavities. We concluded that an essential step is to couple a spin qubit to a photonic-crystal cavity and demonstrate quantum control. The cavity gives a strong interaction between photons and the spin qubit. In the future, this photonic-crystal architecture should be capable of guiding light between two quantum dots.

\section{What are the advantages of using} electron spin in a quantum dot?

Using quantum dots enables us to engineer the system and to control the optical properties more than other systems (for example, nitrogen-vacancy centres in diamond). The other advantage is the ability to perform ultrafast quantum gating of the electron spin. Thanks to the strong dipole moment of quantum dots, optical pulses can initialize and rotate spins very rapidly. This strong coupling to light also gives a strong interaction between quantum-dot transitions and the photonic-crystal cavity. We are hence able to perform quantum gate operations on a picosecond timescale, which is much faster than in other systems.

\footnotetext{
Were there any challenges along the way?

Even though many groups are working to incorporate semiconductor quantum dots in photonic crystals, there has been little progress in combining a spin qubit in a charged quantum dot with a photoniccrystal cavity. So, the first challenge for us was to develop an electrical device to control the charging of the dot that could be incorporated in a thin photonic-crystal membrane. Many parameters needed to be just right to realize a good cavity-quantum-
}

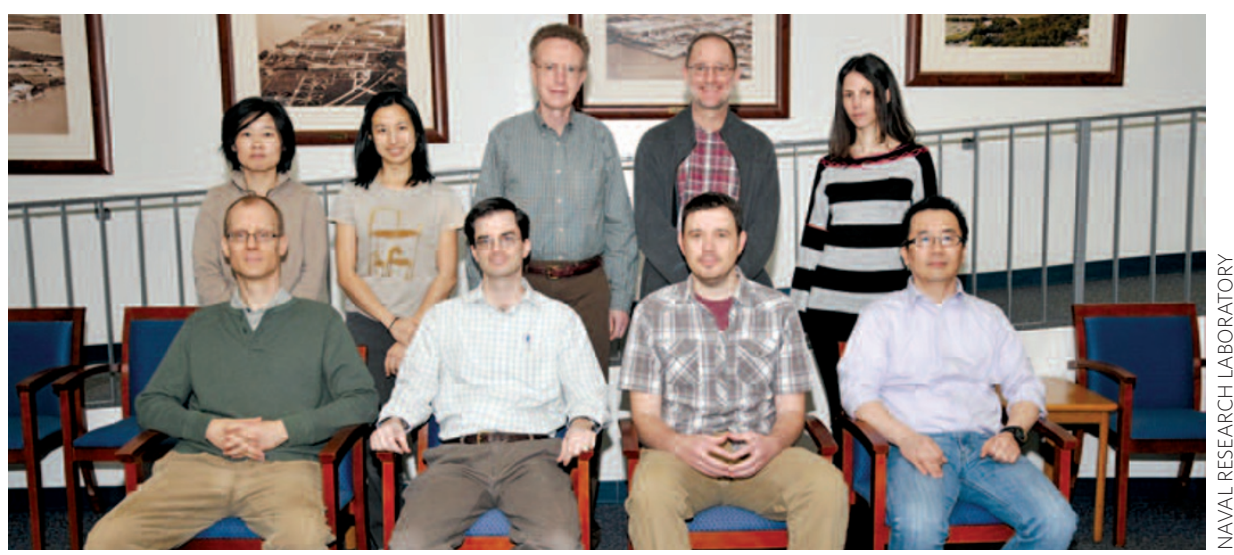

Mijin Kim, Lily Yang, Thomas Reineck, Daniel Gammon and Sophia Economou (top row, left to right) and Allan Bracker, Samuel Carter, Timothy Sweeney and Chul Soo Kim (bottom row, left to right) have successfully produced the interaction between two qubits separated by a significant distance using a photonic-crystal cavity.

dot system, including the cavity quality factor, the spatial and spectral overlap between the cavity mode and the quantum dots, and the polarization properties of the dots and cavity. More recent work has enabled better control; we therefore anticipate finding more systematic ways of creating cavity-quantumdot systems. The second challenge was to learn how to perform quantum control using optical techniques in the photonic-crystal environment. There was significant concern that the linearly polarized cavity mode would prevent optical spin rotation, which relies on circularly polarized pulses. However, we found that pulse detuning can solve this issue.

Were there any unexpected problems? When we charged a single electron in a quantum dot within the photonic crystal, the lifetime of the spin qubit decreased. That's not something we really expected and we are still working on understanding its mechanism. Another issue that came up was the polarization of the quantum dot in the cavity. In a transverse magnetic field, the two sets of optical transitions from one spin state to another have opposite linear polarizations. In some cases these polarizations are aligned with the cavity polarization, whereas in other cases they are not. This polarization alignment has unexpected consequences for initialization and readout of the spin. In the end, we found that one set of transitions is quite useful for fast spin initialization, and the other for spin measurement.

\section{What are its potential applications?} One potential application would be a node in a quantum network. We can transfer quantum information from the spin qubit to a photonic qubit, or perhaps perform on-chip quantum information processing. Another potential application is in classical photonics, where we can use the quantum dots as a switch in a photonic architecture, thereby routing photons from place to place on a chip.

What do you intend to do in the future? One of our goals is to couple two quantum dots in a photonic-crystal environment and actually entangle them using photons. A challenge we face is controlling the system to a high degree: obtaining good spatial and spectral overlap between the dots and the cavity, controlling the polarization axis of the quantum dot, and obtaining higher quality factors.

\section{INTERVIEW BY NORIAKI HORIUCHI} Samuel Carter and colleagues have an Article on quantum control of a spin qubit coupled to a photonic-crystal cavity on page 329 of this issue. 